\title{
Simulation study on the impact of the transmission power on the performance of routing protocols under different Mobility Models
}

\author{
Adam Macintosh \\ Ming FeiSiyau \\ Mohammed Ghavami \\ macintoa@1sbu.ac.uk \\ siyaum@1sbu.ac.uk \\ ghavamim@1sbu.ac.uk \\ BiMEC, Dept. of Engineering \& Design, London South Bank University, London, SE1 0AA, UK
}

\begin{abstract}
- the dynamic topology of a mobile ad hoc network poses a real challenge in designing the routing protocol. This paper examine through simulation the fundamental factors, mobility models and transmission power which have a major impacts on the performance of position based routing protocols. We analyse the effect of the transmission power of on the performance of protocols under two different mobility models. Using OPNET simulation tool, results show the evaluation and performance of the proposed protocol under a unified simulation environment for different scenarios.
\end{abstract}

Keywords - Mobility Models, Mobile Ad hoc networks, Position based routing protocols, Mobility Impacts, OPNET.

\section{INTRODUCTION}

Mobile ad hoc networks (MANETs) is one of the potential upcoming technologies that can support advanced packet services and real-time applications, which also become one of the most innovative and challenging area of wireless networking.

In MANETs, mobile nodes (MNs) operate as routers and end-system connecting points in order to forward packets while moving about, change location frequently and also organize themselves into a temporary 'ad-hoc' network. Because of this, MANETs can offer a larger degree of freedom at a considerably lower cost than other networking solutions. Special routing algorithms are often needed to accommodate changing topology. So far, method for determining the efficient routing paths and delivering messages in an ad hoc environment where the network topology changes has yet to receive much attention. New prototypes are needed to describe the mobile ad hoc feature of wireless networks; and new algorithms are required to effectively and efficiently route data packets to mobile destination in order to support many of multimedia applications. In order to evaluate routing protocol performance. in MANET, the protocol should be tested under realistic conditions on real time basis such as arbitrary obstacles, a sensible transmission range, limited buffer space for the storage of messages, representative data traffic models, and realistic movements of the MNs (i.e. a mobility model)[3,6,17,24].

\section{RELATED WORKS}

Mobility Models (MMs) is the foundation of simulation study on various protocols in MANET. Extensive research has been done in modelling mobility for MANETs and many MMs have been proposed in the literature $[4,7,8,15,22]$.

Comprehensive MMs survey was carried out by Su et al. [13]. A Study by Coroson et al. [1] examined the Routing Protocol Performance Issues and Evaluation Considerations. In this paper, the advantages and limitations of the protocols were examined and expressed as qualitative and quantitative attributes. Paper [15] evaluated the MANET routing protocol AODV under different MMs. In this paper only topology based routing protocols were considered.

Paper by Malarkodi et al. [27] gives a more detailed classification in four categories: temporal dependency, spatial dependency, geographic restriction and hybrid characteristic. In this paper, it emphasises that the results of simulative performance evaluation strongly depends on the models used. Bettstetter et al. [20] examined the spatial node distribution of the random waypoint mobility model. The goal was to define MMs based on motion matrices class and the impact of these metrics on routing performance. Paper [29] investigated throughout simulation the impact of unidirectional link on topology based routing protocols. It indicates, the power control affects the performance of the network layer.

Paper [30] studied the impact of transmission range and Mobility on routing protocols over Ad Hoc. This study was carried out only on topology based routing protocols. Anew routing scheme was proposed to find an optimal path during the routing discovery phase. It is assumed MNs will have alternate 
path to the source in case of failure. It highlighted the results of performance evaluation fluctuate, depending on the models used.

In this research, we investigate through a simulation the impact of the transmission power on the performance of position based routing protocols under different MMs (Dependent and Independent). The impact of transmission power and MMs, on position based routing protocols in MANETs have not been considered before. One of our contributions is investigating the correct adjustment of the MN radio transmission range in order to achieve connected MANETs.

This research also provides practical significance for the simulation study of MANET routing protocols and the design and improvement of MMs. This research is organized as follows. In section 3, a brief description about the positions based routing protocols in our performance evaluation. In section 4, we present the MMs in our performance comparison. In section 5, deeply analyses how the main parameter of the transmission power and MMs, impacts on the performance of routing protocols. In section 6 details of the simulation and results are given. In section 7 the conclusion and future works are discussed.

\section{POSITION BASED ROUTING PROTOCOL}

Position based algorithms overcome the problem related to the maintenance of the routing table in connection oriented algorithms $[2,5,26]$, where the performance degrades quickly when there is an increase in the number of MNs or the speed. Position based routing algorithms eliminate some of the limitations of topology based routing by using geographical information about the $\mathrm{MNs}$ to make decision about routing packets. This position information is obtained by position service and location service. If a MN wants to send data to a destination node, it will make routing decision based on the destination and the positions of the source one-hop neighbours. Consequently, position based routing protocols do not require route establishment or maintenance. Position information only needs to be distributed in the local area.

\section{A. Greedy Perimeter Stateless Routing}

Greedy Perimeter Stateless Routing (GPSR) proposed by Karp and Kung is a position based routing algorithm [8]. GPSR makes greedy forwarding decisions using only information about the position of immediate neighbours in the network topology. Packets are forwarded to the next-hop node which moves the packet to a nodes which most close to the position of the destination.

By keeping only local topology information, GPSR scales better than topology based routing as the number of network destinations increases. If the packet reaches a region where greedy forwarding is impossible, the algorithm enters into recovery mode by routing around the perimeter of the region $[1,4,8,19]$. The GPSR protocol is a routing protocol that is often used to establish routes in MANET or sensor networks.

However, for it to operate effectively, it is a requirement that all MNs assist each other. However, such a process would be unlikely to perform efficiently in MANET. The disadvantages of GPSR are the control overhead and slow recovery process [4, $8,17,25]$.

\section{B. Local Area Dynamic Routing protocol}

In the previous work, Local area dynamic routing protocol (LANDY) $[10,14]$ localises routing information distribution in the one-hop range. Thus LANDY Fig.1 will reduce the control overhead, simplify routing computation and save memory storage. Each $\mathrm{MN}$ in the network needs to maintain the local status of its MNs neighbours only. For each connection, a MN gets order of query packets $(\mathrm{Ni})$. The number of neighbour MNs (Ni) may increase or decrease based on the movement of MNs within the local region. Therefore the distribution of the MNs within a region for the network state is $\mathrm{S}(\mathrm{n})$ in the worst case scenario.

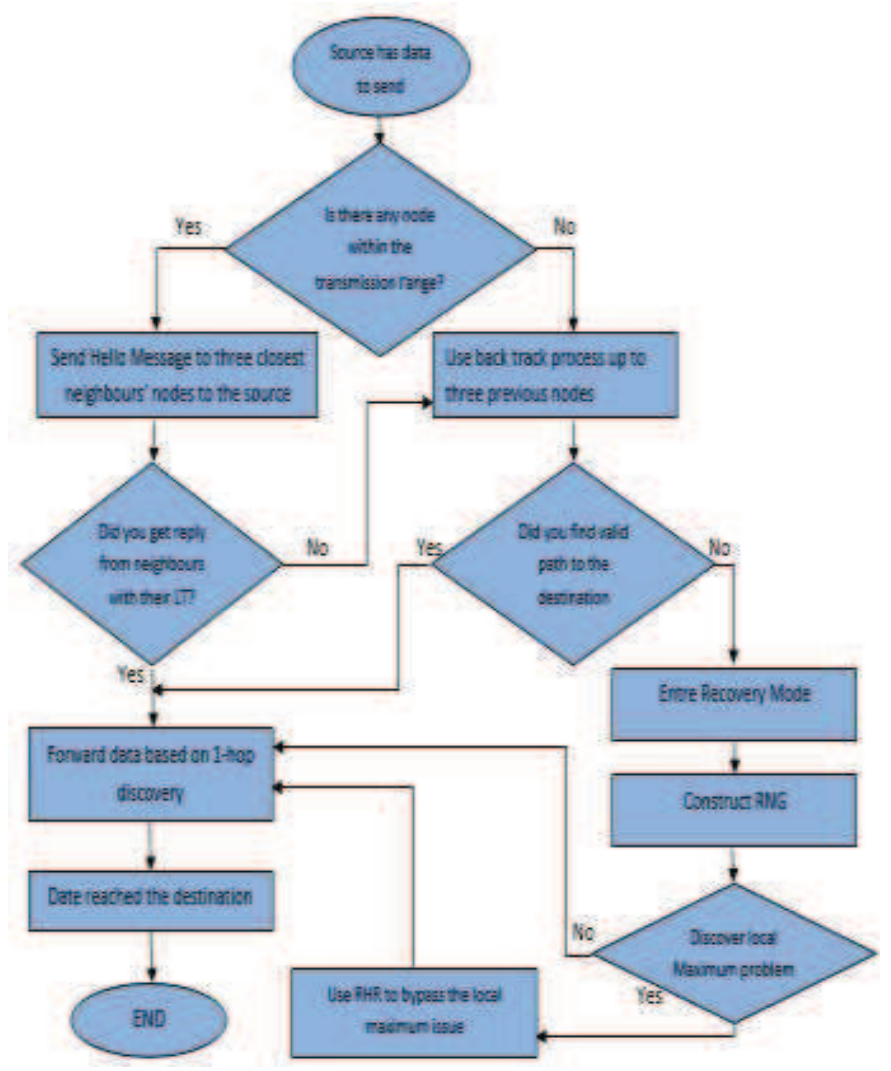

FIG 1. LANDY ROUTING PROTOCOLS

The MN updates its locomotion components (LC) through position service (e.g. GPS) periodically in LANDY. The MN broadcasts its Mobile code identifier (MCID), Cell code identifier (CCID) and LC in a HELLO message periodically. Data packets are marked with the LC of the sender and the destination, so that the receiving nodes are able to update the neighbour's locomotion information upon receiving the data packet. The MN does not flood the HELLO message. Thus, the LANDY routing protocol reduces the control overhead and simplifies the routing computation. The HELLO message broadcasting mechanism makes all nodes aware of their neighbours' locomotion information. Each MN periodically 
broadcasts a HELLO message to its one-hop neighbours, with its MCID, CCID and LC. The HELLO message inter-arrival time is jittered with a uniform distribution to avoid synchronization of neighbours' HELLO messages that could result in conflict. Each MN updates its locomotion table (LT) of neighbours when it receives a HELLO message.

The LT associates an expiration value with each entry. If the node does not receive a HELLO message from a neighbour within the expiration time, it removes the neighbour from the table.

\section{MOBILITY MODELS IN MANET}

MMs designed to represent the motion of MNs, and how their location, velocity, acceleration changes over time. MMs used to evaluate the performance of ad hoc network protocols. Since the performance of protocol depends on the mobility model, it is important to choose a suitable model for the evaluated protocol. Generally, there are two types of MMs used in the simulation of wireless networks; Independent - Entity Mobility Models (IEMMs) and Dependent - Group Mobility Models (DGMMs). In IEMMs a node's movement does not control in anyway, other nodes' movements. Nodes move independently from each other, randomly. i.e. Random Waypoint Model, Random Walk Model, Random Direction Model, Gauss-Markov model, Manhattan Mobility Model.

DGMMs Represent MNs whose movements are mutually dependent on the group movement. DGMMs used when MNs cooperate with each other to accomplish a common goal. Typical situations do exist in military environments (soldiers move together), i.e. Reference Point Group Model, Nomadic Community Model, Column Mobility Model, Pursue Mobility Model.

\section{A. Gauss-Markov model}

Gauss-Markov model (GMM) is a model that uses one tuning parameter to vary the degree of randomness in the mobility pattern. GMM was designed to adapt to different levels of randomness via tuning parameters $[3,15]$. GMM is a different model from Random Waypoint in terms of velocity management. In this model, the velocity of $\mathrm{MN}$ is correlated over time and GMM random process. GMM random process satisfies the requirements for both Gaussian processes and Markov processes. The velocity of $\mathrm{MN}$ at time slot $\mathrm{t}$ is dependent on the velocity at time $(\mathrm{t}-1)$.

Therefore, GMM is a dependent mobility model where the dependency is determined by the parameter which affects the randomness of GMM process. By tuning this parameter, different mobility model can be created $[20,21]$.

GMM creates movements, which are dependent on node's current speed and direction. The idea is to eliminate the sharp and sudden turns present in the Random Waypoint even by keeping a certain degree of randomness.

\section{B. Reference Point Group Model}

Reference Point Group Model (RPGM) represents the random movement of a group of MNs as well as the random movement of each individual MN within the group. RPGM is a group mobility model where group movements are based after the path travelled by a logical centre. RPGM used to calculate group motion via a group motion vector, group mobility. The movement of the group centre completely describes the movement of this corresponding group of MNs. Including their direction and speed. Individual $\mathrm{MNs}$ randomly move about their own predefined reference points whose movements depend on the group movement.

\section{ANALYSIS ON IMPACT OF TRANSMISSION POWER AND MOBILITY MODELS}

In order to explain how the transmission power and MMs impact on the performance of the MANET routing protocols, various predominance metrics used and performance discrepancies analysed in this section.

\section{A. Simulation setup}

We have chosen LANDY [10], and GPSR [8] position based MANET routing protocols for performance investigation under different MMs. Both protocols were evaluated under GMM and RPGM using Optimized Network Engineering Tools (OPNET) v14.5.

OPNET is a well-established and highly professional product.

It has being developed for almost 20 years. It is broadly held to be the state-of-the-art in network simulation. It has a lots of features and toolsets containing a packet format that defines protocols, a node model for specifying network component interface, a process model for concept of behavior of a specific network component, a project window for defining the topology of the network and several linkages, and a simulation window that is able to capture and/or display the results of network simulation.

The MMs are computed using C-code programs, whose results are imported into OPNET simulation models. Each node is then assigned a particular trajectory. The LANDY protocol is implemented in the OPNET as a process model in wireless MNs. The LANDY process model can be represented in a State Transition Diagram (STD). MN models were constructed that included OPNET standard IEEE 802.11 physical and MAC layers, as well as custom build process models to implement the LANDY protocol.

The traffic application is a traffic generator. This traffic generator starts at 10 s during simulation. Every model has the mean speed changing from $10 \mathrm{~m} / \mathrm{s}$ to $30 \mathrm{~m} / \mathrm{s}$ with zero pause time. In all this patterns, 100 nodes move in an area of $1000 \mathrm{~m} \times$ $1000 \mathrm{~m}$ for a period of $1200 \mathrm{~s}$, to avoid the effect of initializing and ending, we only gather the data between $100 \mathrm{~s}-1100 \mathrm{~s}$. The error bars indicate $95 \%$ confidence intervals in all the scenarios. 
Six sets of source and destination pairs were selected randomly from a group of $100 \mathrm{MNs}$. Constant bit rate (CRB), used to set the rate of the transmitted data packet, which is set to 8 packets /s, and the size of user datagram packet (UDP) is fixed to 512 bytes. The accurate adjustment of the MNs radio transmission power is key factor in the simulation. It allows the controlling of the network topology in MANET [9,30].If we increase the transmission power of a $\mathrm{MN}$, this will result in higher range and consequently reach more MNs via a direct link. Otherwise if we set the power low, this might result in isolation without any link to other MNs.

We have configured the six sets with two different power levels Table 1. Each set will cover various volume of unidirectional links. For example, set 0.1 represents $10 \% \mathrm{MN}$ with low transmission range and $90 \%$ with high transmission range. This method will aid the performance investigation for scenarios with various volume of unidirectional links.

Table 1: Ratio set for unidirectional links

\begin{tabular}{|l|c|c|c|c|c|c||}
\hline Set No. & Set & Set & Set & Set & Set & Set \\
& 0 & 0.1 & 0.2 & 0.3 & 0.4 & 0.5 \\
\hline No. of MNs & 0 & 20 & 40 & 60 & 80 & 100 \\
\hline
\end{tabular}

The high level is assigned to $\mathrm{MN}$ with transmission range 300 $\mathrm{m}$, and the low level is assigned to $\mathrm{MN}$ with $125 \mathrm{~m}$ transmission range. Due to the dynamic topology of the MNs, it is not possible to determine the exact number of links, which results in route repeatedly being assembled and breaks. The MAC radio propagation bit rate is set to $11 \mathrm{Mb} / \mathrm{s}$ with frequency operating at $2.422 \mathrm{GHz}$. Table 2. represent the setting for MMs on both protocols.

Table 2: Configuration parameters of mobility models

\begin{tabular}{||l|l|l||}
\hline Parameter & GMM & RPGM \\
\hline No. of Mobile Nodes & 100 & 100 \\
\hline Speed update frequency & $2.5 \mathrm{~s}$ & NA \\
\hline Angle std deviation & 450 & NA \\
\hline Speed std deviation & $1.5 \mathrm{~m} / \mathrm{s}$ & NA \\
\hline Group deviation & NA & 2 \\
\hline Pause time & NA & $0 \mathrm{~s}$ \\
\hline No. of groups & NA & 10 groups \\
\hline \hline
\end{tabular}

\section{B. Simulation results}

The unidirectional links results are shown in Fig. 1, Fig. 2, Fig. 3 and Fig. 4 for LANDY, GPSR as a function of radio range in the 100-node scenarios, respectively. The result indicates that at higher speed, the probability of unidirectional links occurrences is higher.

Routes between the MN become unstable at higher speed, due to the dynamic topology and possibly break, leading to unidirectional links. The results shows that GMM generate more unidirectional links compared to RPGM on both protocols. At speed of $0 \mathrm{~m} / \mathrm{s}$ crossing set 0 Fig.1, Fig.2, Fig.3and Fig.4, on both protocols, we have noticed a small number of unidirectional links generated. Due to the interfering by neighbour MNs which result in packet dropping. Also, with increasing the speed of the MNs, this will lead to link breaks frequently and resulting to interpretation as unidirectional links by both routing protocols. When the number of unidirectional links fluctuate at high rate mobility rate, the slight drop is due to the fact that the number of RREQ "Route Request" packet sent by the source node decreases, and it indicates that either the routing paths has been successfully constructed, or there exists more bidirectional links in the network than the unidirectional links. Also, low transmission range does not always provide an increase in number of unidirectional link, due to the impact of other factors such as the behavior of mobility model and speed MNs.

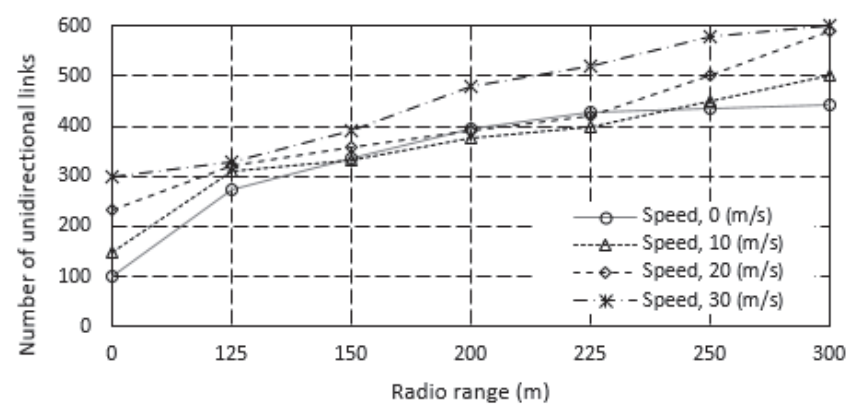

Fig. 1 GMM unidirectional links vs Radio range - LANDY

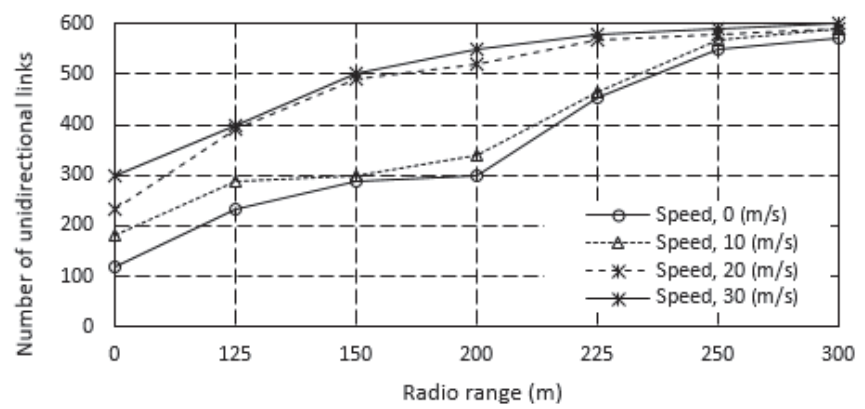

Fig. 2 RPGM unidirectional links vs Radio range - LANDY

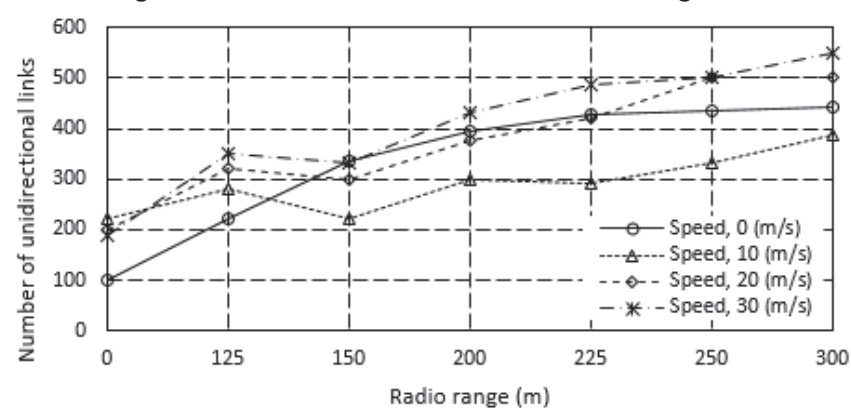

Fig. 3 GMM unidirectional links vs Radio range - GPSR 


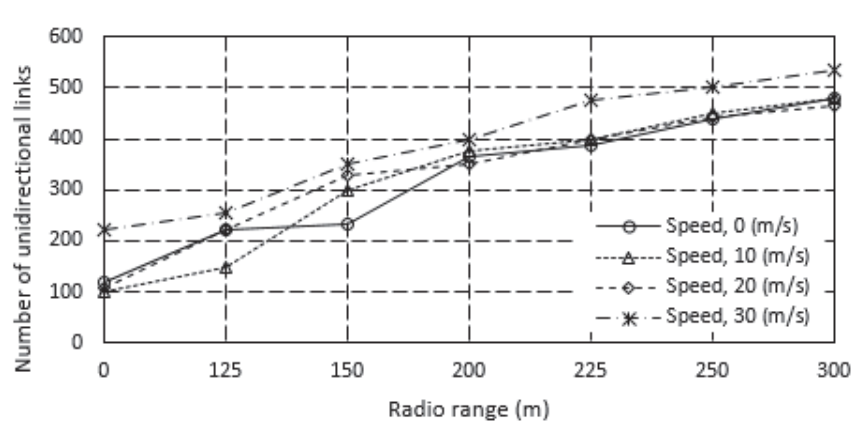

Fig. 4 RPGM unidirectional links vs Radio range - GPSR

The results of the average RREQ packet sent by each source MNs are shown in Fig. 5, Fig. 6, Fig. 7 and Fig. 8 for LANDY, GPSR as a function of radio range in the 100-node scenarios, respectively. The source MNs send RREQ at route discovery and recovery process of route failure on both routing protocols. Results indicates that, the higher mobility of MNs result in increasing the production of RREQ in the network. Which causes routing overhead. With speed increasing more over head is generating in both protocols. But LANDY have less overhead than GPSR. Also, by observing more simulation experiments, shows that more than $80 \%$ of routing packets in the network is created by the RREQ packet of MNs.

In general, the performance of GPSR drops with increasing number of nodes set with low transmission range, but LANDY perform well comparing to GPSR. Results also shows that, the impact of RPGM on routing performance is minimal, compared with GMM. Such performance is due to MNs closeness, which restricts movement to within a small area around the reference point. As a result, link connectivity increases, leading to less unidirectional links occurrences. On the other hand, MNs in GMM are uniformly distributed. Consequently, nodes are more vulnerable to form unidirectional link.

In addition, result shows with the speed increasing, each metrics is getting worse in some way. These results exist since the topology of the network is more unstable with the speed increasing. As a result of the RPGM model only has pause time in simulation boundary and the MNs need to keep moving in the same direction until they reach the border of the simulation area. The metric in RPGM model is better than that of GMM model.

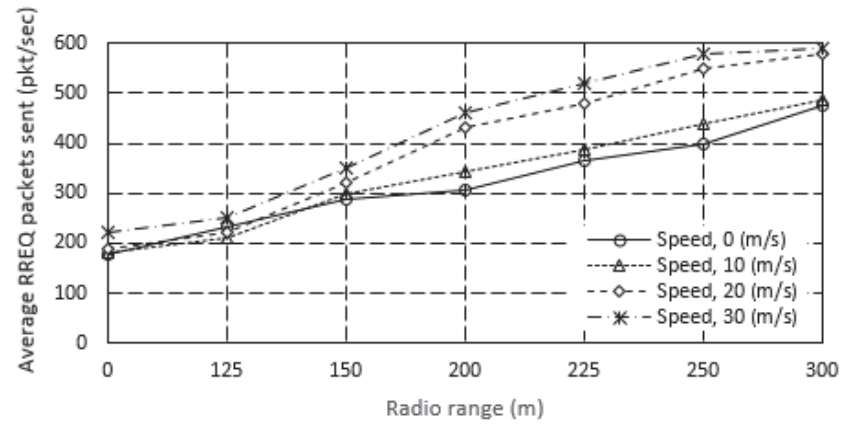

Fig. 5 GMM Average RREQ packet sent vs Radio range - LANDY

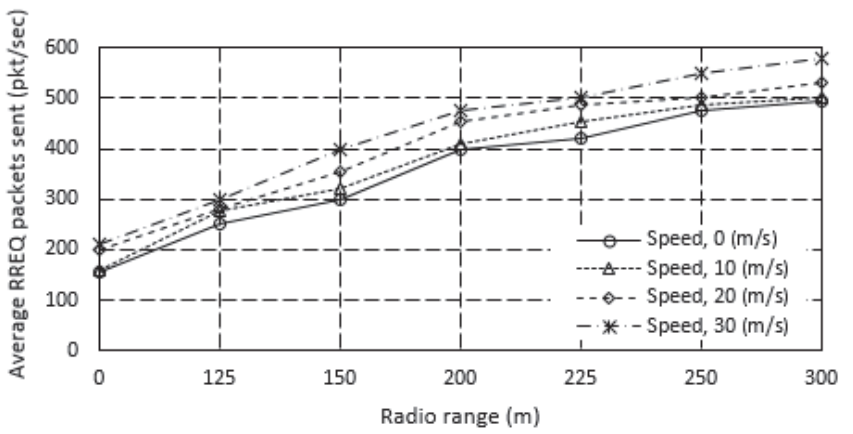

Fig. 6 RPGM Average RREQ packet sent vs Radio range - LANDY

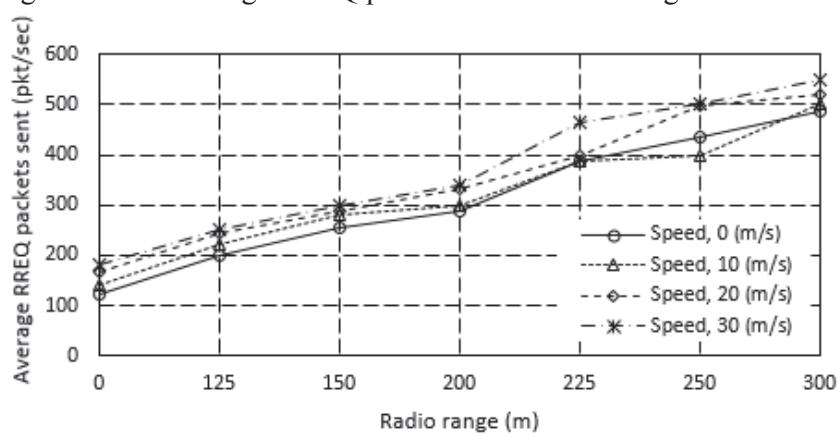

Fig. 7 GMM Average RREQ packet sent vs Radio range - GPSR

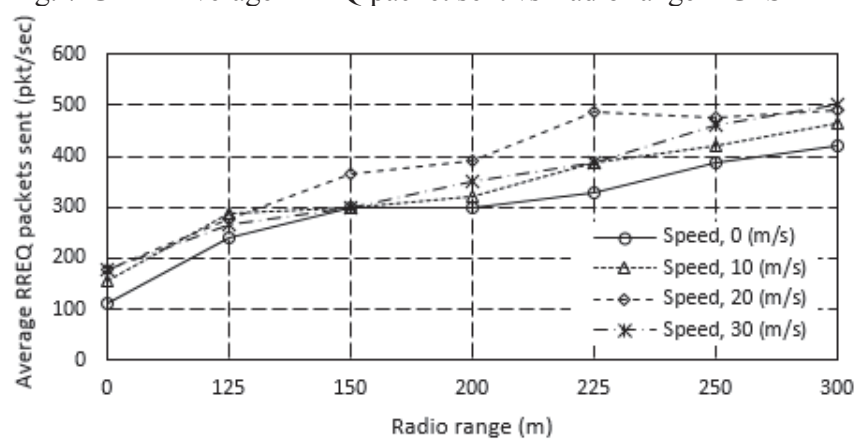

Fig. 8 RPGM Average RREQ packet sent vs Radio range - GPSR

\section{CONCLUSIONS AND FUTURE WORK}

The effects of the transmission power, and MMs on the performance metric of MANET routing protocols have been analysed. The simulation results indicate that even setting the same parameters, different MMs have a different impact on the performance evaluation of protocols. In contrast, the RPGM model provides some accurate motion with expectable speed and turning angle. Therefore, choosing an appropriate mobility model as well as setting appropriate parameters serve as the key role for protocol evaluation. It is found that Protocols that have link layer support for link breakage detection, are much more stable.

The performance of the protocols differs slightly during different network loads. A higher sending rate causes the protocol to detect broken links faster, thus reacting faster; this leads to a slight increase in control packets, which affects the byte overhead. The increased send rate also sets demands on the send buffer of the routing protocol. 
A tremendous amount of research remains to be done in the area of MMs in ad hoc networks. Group Pursuit Models are of special interest for future compact systems "FCS" applications, and have to be included in a comprehensive simulation.

\section{REFERENCES}

[1] S. Corson and J. Macker, "Mobile Ad hoc Networking (MANET): Routing Protocol Performance Issues and Evaluation Considerations", RFC: 2701, January 1999.

[2] C. Bettstetter. "Smooth is better than sharp: A random mobility model for simulation of wireless networks", In: Proceedings of 4th ACM International Workshop on Modeling, Analysis and Simulation of Wireless and Mobile Systems(MSWiM'01), Rome, Italy, 2001, 07: 19-27.

[3] T. Camp, J. Boleng, V. davies, "A Survey of Mobility Models for Ad Hoc Network Research", Wireless Communication \& Mobile Computing(WCMC): Special issue on Mobile Ad Hoc Networking: research.Trends and Applications, 2002, 2(5)-483-702.

[4] C. Lal, V. Laxmi, M. Gaur "A Node-Disjoint Multipath Routing Method Based on AODV Protocol for MANETs", In Proceeding(s) of (IEEE international conference) Advanced Information Networking and Applications (AINA), pp. 399-405, 2012.

[5] V. Lenders, J. Wagner, and M. May, "Analyzing the Impact of Mobility in Ad Hoc Networks", In ACM REALMAN, Florence, Italy. May 2006.

[6] X. Xiang , Z. Zhou and X. Wang "Self-Adaptive On Demand Geographic Routing Protocols for Mobile Ad Hoc Networks", Proc. IEEE INFOCOM, 2007.

[7] M. Xu, Q. Wu, G. Xie, Y. Zhao, "The impact of mobility models in mobile IP multicast research," International Journal of Ad Hoc and Ubiquitous Computing, vol. 4, no.3, pp. 191 - 200, 2009.

[8] B. Karp, H. T. Kung, "GPSR: greedy perimeter stateless routing for wireless networks", Proceedings of the 6th annual international conference on Mobile computing and networking, p.243-254, August 06-11, 2000, Boston, Massachusetts, USA.

[9] F. Bai, N. Sadagopan and A. Helmy, "IMPORTANT: A framework to systematically analyze the Impact of Mobility on Performance of Routing protocols for Ad hoc Networks," in IEEE INFOCOM, March 2003

[10] A. Macintosh, M. Ghavami, and M. FeiSiyau"Lightweight Local Area Network Dynamic Routing Protocol for MANET ", International Journal of Soft Computing and Software Engineering [JSCSE], Vol. 2, No. 7, pp. 9-25, 2012, Doi: 10.7321/jscse.v2.n7.2.

[11] M. J. B. Appel and R. P. Russo, "The minumum vertex degree of a graph on uniform points in [0,1]d," Adv. in Applied Probability, vol. 29, pp. 582-594, 1997.

[12] P. Gupta, R. Drag, an experimental scaling law for ad hoc networks, Bell Laboratories Technical report. Bell Laboratories, Murray Hill 2006.

[13] F. Akyildiz, W. Su, et. al., "A Survey on Sensor Networks", IEEE Communication Magazine, August 2002, pp. 102-114.

[14] A. Macintosh, M. Ghavami, and M. FeiSiyau "Local Area Network Dynamic (LANDY) routing protocol: A position based routing protocol for MANET", IEEE European Wireless. EW 18th European Wireless Conference, May 2012.

[15] C. Perkins, E. Royer and S. Das, "Ad hoc On-Demand Distance Trajectory,(AODV)routing”IETF,RFC3561:http://tools.ietf.org/html/rf c3561 (July 2003).

[16] Sheikhan, M, Hemmati, E. " High reliable disjoint path set selection in mobile ad-hoc network using hopfield neural network", journal of Communications, IET, vol. 5, no. 11, pp. 1566 - 1576, 2012.

[17] H. Asenov and V. Hnatyshin, "GPS-Enhanced AODV routing", Proc. 2009 International Conference on Wireless Networks (ICWN'09), Las Vegas, NV, 2009

[18] F. Bai, N. Sadagopan, B. Krishnamachari and A. Helmy, "Modeling path duration distributions in MANETs and their impact on reactive routing protocols," IEEE Journal on Selected Areas in Communications, vol. 22, no. 7, pp. 1357-1373, September 2004..

[19] T. Jun, A. Dalton and S. Bodas, "Expressive analytical model for routing protocols in mobile ad hoc networks," Proc. IEEE International Conference on Communications (ICC 2008), pp. 2134-2140, Beijing, China, May 2008

[20] C. Bettstetter and C. Wagner, "The spatial node distribution of the random waypoint mobility model", in Proc. 1st German Worksh. Mob. Ad-Hoc Netw. WMAN'02, Ulm, Germany, 2002, pp. 41-58.

[21] H. Rangarajan and J.J. Garcia-Luna-Aceves. Using Labeled Paths for Loop-free On-Demand Routing in Ad Hoc Networks. in ACM MobiHoc 2004. May 24--26, 2004. Tokyo, Japan.

[22] R. Bai and M. Singhal, "DOA: DSR over AODV routing for mobile adhoc networks," IEEE Transactions on Mobile Computing, no. 14 03-1416, 2006.

[23] H. Takahi and L. Kleinrock, "Optimal transmission ranges for randomly distributed packet radio terminals," IEEE Trans. on Comm., Mar. 2007.

[24] W. Kellerer, C. Bettstetter, C. Schwingenschl“ogl,P. Sties, K.-E. Steinberg, and H.-J. V"ogel, "(Auto) Mobile communication in a heterogeneous and converged world," IEEE Pers. Comm., Dec. 2001.

[25] T. K. Philips, S. S. Panwar, and A. N. Tantawi, "Connectivity properties of a packet radio network model," IEEE Trans. on Inform. Theory, Sept. 1998.

[26] L. Blazevic, J.-Y. L. Boudec, and S. Giordano, A location -based routing method for mobile ad hoc networks," IEEE Trans. Mobile Computing, vol. 4, no. 2, pp. $97\{110$, Mar. 2005

[27] B. Malarkodi., P.Gopal,B.Venkataramani, "Performance Evaluation of Adhoc Networks with Different Multicast Routing Protocols and Mobility Models," International Conference on Advances in Recent Technologies in Communication and Computing, pp.81-84, 2009.

[28] I. Stepanov, and K. Rothermel, "On the impact of a more realistic physical layer on MANET simulations results," Ad Hoc Networks, Vol. 6, no. 1, pp. 61-78, Jan. 2008.

[29] M. Zuhairi, D. Harle, A simulation study on the impact of mobility models on routing protocol performance with unidirectional link presence, International Conference on Information Networking, 2011.

[30] R. Kumar Gujral, J. Grover, A. Anjali, S. Rana, Impact of Transmission Range and Mobility on Routing Protocols over Ad Hoc Networks, International Conference on Computing Sciences, 2012 